\title{
Optimal and Robust Controllers for Periodic and Multirate Systems
}

\author{
Munther A. Dahleh, Member, IEEE, Petros G. Voulgaris, and Lena S. Valavani, Member, IEEE
}

\begin{abstract}
In this paper, the problem of optimal rejection of bounded persistent disturbances is solved in the case of linear discrete-time periodic systems. The solution consists of solving an equivalent time-invariant standard $l^{1}$ optimization problem subject to an additional constraint. This constraint assures the causality of the resulting periodic controller. By the duality theory, the problem is shown to be equivalent to a linear programming problem, which is no harder than the standard $l^{1}$ problem. Also, it is shown that the method of solution presented applies exactly to the problem of disturbance rejection in the case of multirate sampled data systems. Finally, we apply the results to the problem of robust stabilization of periodic and multirate systems.
\end{abstract}

\section{INTRODUCTION}

$\mathrm{T}$ HE study of periodically time-varying systems is a topic of growing research. In [9] an equivalence between $m$-input $p$-output linear $N$-period causal discrete systems and a class of discrete linear time-invariant causal systems was established. Namely, this class consists of $m N$-input $p N$ output linear time-invariant (LTI) systems with $\lambda$-transforms $\hat{P}(\lambda)$ such that $\hat{P}(0)$ is a block lower triangular matrix. This equivalence is strong in the sense that it preserves the algebraic structure (isomorphism) and the norm (isometry). Hence, we can effectively use the theory of LTI systems to study periodic ones. In fact, the authors in [9] use this equivalence to prove that although the performance is not improved, periodic compensators for LTI plants offer significant advantages in terms of robustness to parametric uncertainty. Moreover, they argue that the optimal (in $l^{2}$ to $l^{2}$ sense) compensator for an $N$-periodic system is $N$-periodic. Indeed, as it is proved in [1] the above argument is true also in the worst case $l^{\infty}$ to $l^{\infty}$ sense. Hence, it can be easily inferred that the optimal controller for the $\mathrm{N}$-periodic system can be obtained by solving the equivalent LTI problem. This problem however, includes a constraint on the optimal LTI compensator $\hat{C}(\lambda)$, namely, $\hat{C}(0)$ should be block lower triangular matrix so that $C$ corresponds to a causal $N$-periodic controller. It is exactly this problem we approach in this paper in an optimal $l^{\infty}$ to $l^{\infty}$ sense. The unconstrained

Manuscript received December 10, 1990; revised June 12, 1991. Paper recommended by Associate Editor, J. S. Freudenberg. The work of M. A. Dahleh was supported by the Army Research Office under Grant DAAL-86K-0171 and by the National Science Foundation under Grant 8810178-ECS. The work of P. G. Voulgaris and L..S. Valavani was supported by the Air Force Office on Scientific Research, Eglin Air Force Base, under Grant F08635-87-K-0031, by the NASA Ames and Langley Research Centers under Grant NASA/NAG-2-297 and by a gift from the Boeing Corporation.

The authors are with the Laboratory of Information and Decision Systems, Massachusetts Institute of Technology, Cambridge, MA 02130.

IEEE Log Number 9104613. problem is solved in [2], [3]. In [2], [3] the problem is transformed to a tractable linear programming problem, via duality theory. In this paper, we show that the same approach can be extended to yield the optimal solution for the constrained problem. In Section II, we present some mathematical preliminaries together with some background on periodic systems. In Section III, the problem is defined and in Section IV, we present the solution following two approaches. In Section V, we demonstrate that the problem of optimal disturbance rejection for multi-rate sampled systems can be treated analogously. In particular, we show how with a simple modification the same approach can be used to obtain the optimal multirate compensator. Furthermore in the same section, we consider the problem of robust stabilization in periodic and multirate plants. We indicate that this problem can be analyzed without introducing conservatism by considering the same problem for the equivalent LTI system. Finally, in Section VI we summarize and draw conclusions.

\section{Notation ANd Preliminaries}

In this paper the following notation is used:

$l_{m \times n}^{1} \quad$ The normed linear space of all $m \times n$ matrices $H$ each of whose entries is a right sided, absolutely summable real sequence $H_{i j}=$ $\left(H_{i j}(k)\right)_{k=0}^{\infty}$. The norm is defined as

$$
\|H\|_{l_{m \times n}^{1}}=\max _{i} \sum_{j=1}^{n} \sum_{k=0}^{\infty}\left|H_{i j}(k)\right| .
$$

$\mathscr{A}_{m \times n}^{*} \quad$ The normed linear space of all $m \times n$ matrices $H$ each of whose entries is a right sided magnitude bounded real sequence $H_{i j}=\left(H_{i j}(k)\right)_{k=0}^{\infty}$. The norm is defined as

$$
\|H\|_{\mathscr{A}_{m \times n}^{*} \times n}=\sum_{i=1}^{m} \max _{j}\left(\sup _{k}\left|H_{i j}(k)\right|\right) .
$$

$c_{m \times n}^{0} \quad$ The subspace of $\mathscr{A}_{m \times n}^{*}$ consisting of all elements which converge to zero.

$l_{m}^{\infty} \quad$ The space of real $m \times 1$ vectors $u$ each of whose components is a magnitude bounded real sequence $\left(u_{i}(k)\right)_{k=0}^{\infty}$. The norm is defined as

$$
\|u\|_{l_{m}^{\infty}}=\max _{i}\left(\sup _{k}\left|u_{i}(k)\right|\right) .
$$

$l_{m}^{\infty, e} \quad$ The space of real $m \times 1$ vector valued sequences.

$\hat{H}(\lambda) \quad$ The $\lambda$-transform of a right sided $m \times n$ real 
sequence $H=(H(k))_{k=0}^{\infty}$ defined as

$$
\hat{H}(\lambda)=\sum_{k=0}^{\infty} H(k) \lambda^{k} .
$$

$\mathscr{A}_{m \times n} \quad$ The real normed linear space of all $m \times n$ matrices $\hat{H}(\lambda)$ such that $\hat{H}(\lambda)$ is the $\lambda$-transform of an $l_{m \times n}^{1}$ sequence $H$. This space is isometrically isomorphic to $l_{m \times n}^{1}$.

$\mathscr{L}_{T V}^{m \times n}$ The space of all linear bounded and causal maps from $l_{n}^{\infty}$ to $l_{m}^{\infty}$. We refer to these operators as stable.

$\mathscr{L}_{T I}^{m \times n} \quad$ The subspace of $\mathscr{L}_{T V}^{m \times n}$ consisting of maps that commute with the shift operator (i.e., the timeinvariant maps). This space is isometrically isomorphic to $\mathscr{A}_{m \times n}$.

$X^{*} \quad$ The dual space of the normed linear space $X$.

$B X \quad$ The closed unit ball of $X$.

${ }^{\perp} S \quad$ The left annihilator of $S \subset X$.

$S^{\perp} \quad$ The right annihilator of $S \subset X$.

$\left\langle x, x^{*}\right\rangle$ The value of the bounded linear functional $x^{*}$ at point $x \in X$.

$\Pi_{m}^{k} \quad$ The $k$ th-truncation operator on $l_{m}^{\infty, e}$ defined as

$\Pi_{m}^{k}:\{u(0), u(1), \cdots,\} \rightarrow\{u(0), \cdots, u(k), 0,0, \cdots$,$\} .$

$\Lambda_{m} \quad$ The right shift operator on $l_{m}^{\infty, e}$, i.e.,

$$
\Lambda_{m}:\{a(0), a(1), \cdots,\} \rightarrow\{0, a(0), a(1), \cdots,\} .
$$

Note: We will often drop the $m$ and $n$ in the above notation when the dimension is not important or when it is clear from the context. Also, subscripts on the norms are dropped when there is no ambiguity.

We now present some facts that are used in this paper. First, we invoke the following from [2].

Fact 2.1: Every linear functional on $l_{m \times n}^{1}$ is representable uniquely in the form

$$
f(H)=\sum_{i=1}^{m} \sum_{j=1}^{n} \sum_{k=0}^{\infty} Y_{i j}(k) H_{i j}(k)
$$

where $Y=\left(Y_{i j}\right) \in \mathscr{A}_{m \times n}^{*}$ and $H=\left(H_{i j}\right) \in l_{m \times n}^{1}$. Hence, $\left(l_{m \times n}^{1}\right)^{*}=\mathscr{A}_{m \times n}^{*}$. It can be also shown that $\left(c_{m \times n}^{0}\right)^{*}=l_{m \times n}^{1}$ where the linear functionals are defined as above.

Next, we give some background on periodic systems following [9].

Definition 2.1: Let $f: l_{m}^{\infty, e} \rightarrow l_{n}^{\infty, e}$ be an operator. $f$ is called causal if

$$
\Pi_{n}^{k} f u=\Pi_{n}^{k} f \Pi_{m}^{k} u, \quad \forall k=0,1,2, \cdots,
$$

$f$ is called strictly causal if

$$
\Pi_{n}^{k} f u=\Pi_{n}^{k} f \Pi_{m}^{k-1} u, \quad \forall k=0,1,2, \cdots .
$$

Now let $f$ represent the input-output map from $l_{m}^{\infty, e}$ to $l_{p}^{\infty, e}$ of a linear causal time-varying system.

Definition 2.2: The map $f$ is $N$-periodic if and only if it commutes with the $N$ th power of the right shift, i.e.,

$$
f\left(\Lambda_{m}\right)^{N}=\left(\Lambda_{p}\right)^{N} f
$$

Let $f$ be $N$-periodic and $W_{m}$ represent the isomorphism

$$
\begin{gathered}
W_{m}: l_{m}^{\infty, e} \rightarrow l_{m N}^{\infty, e} \\
a=\{a(0), a(1), \cdots,\} \rightarrow W_{m}(a) \\
=\left\{\left(\begin{array}{c}
a(0) \\
a(1) \\
\vdots \\
a(N-1)
\end{array}\right),\left(\begin{array}{c}
a(N) \\
a(N+1) \\
\vdots \\
a(2 N-1)
\end{array}\right), \cdots,\right\}
\end{gathered}
$$

Define the map $L$ as

$$
L(f)=W_{p} f W_{m}^{-1}
$$

where $W_{p}$ is defined similarly to $W_{m}$. Then $L(f)$ represents a system with inputs in $l_{m N}^{\infty, e}$ and outputs in $l_{p N}^{\infty}$. . Moreover, as shown in [9], $L(f)$ is LTI and the following hold.

Fact 2.2: Given a $m$-input $p$-output linear causal $N$-periodic system $f$, one can associate via the map $L$ a unique causal $(p N \times m N)$ LTI system $L(f)$ with a transfer matrix $\hat{F}(\lambda)$. Conversely, any $(p N \times m N)$ transfer matrix $\hat{F}(\lambda)$ with $\hat{F}(0)$ block lower triangular can be associated by $L^{-1}$ with a unique $m$-input $p$-output linear causal $N$-periodic system.

Note: In the above fact, $\hat{F}(0)$ block lower triangular means that

$$
\hat{F}(0)=\left(\begin{array}{ccccc}
F_{00} & 0 & 0 & \cdots & 0 \\
F_{10} & F_{11} & 0 & \cdots & 0 \\
\vdots & \vdots & \vdots & \ddots & 0 \\
F_{N 0} & F_{N 1} & F_{N 2} & \cdots & F_{N N}
\end{array}\right)
$$

where each submatrix $F_{i j}$ has a dimension $p \times m$. This is exactly what is meant when the term block lower triangular is used hereafter. Also, we will use the term "lifting" to indicate the action of $L$ to a system $f$ (i.e., $F$ is the lifted system or $F$ is the lift of $f$ ). Finally, to avoid proliferation of notation, we hereafter use $W$ generically instead of $W_{n}$ where the subscript $n$ specifies the dimension of the elements of the sequence that this isomorphism acts on.

Fact 2.3: $L$ preserves the algebraic properties and the norm. In particular

$$
\sup _{u \in B l_{m}^{\infty}}\|f u\|_{l_{p}^{\infty}}=\sup _{w \in B l_{m N}^{\infty}}\|F w\|_{l_{p N}^{\infty}} .
$$

Hence, $f$ is input-output stable if and only if $F=L(f)$ is stable.

Suppose in addition, that $f$ is finite dimensional with a stabilizable and detectable state-space description. In [1], [13] it is shown that we can obtain a doubly coprime factorization (DCF) of $f$ by obtaining a DCF of the lifted system $F$. The key observation in [1] is that the factors of $F$ obtained using the standard formulas in [7] possess the property of being block lower triangular at $\lambda=0$. Hence, since $L$ is an isomorphism, we can obtain a DCF of $f$, the factors being the images of the inverse map of the lifted LTI factors of $F$ and therefore $N$-periodic. Also assuming wellposedness [7], [5] we can characterize all stabilizing con- 
trollers $c$ in terms of these factors, In summary, we have the following.

Fact 2.4: Let $F=N_{l} D_{l}^{-1}=D_{r}^{-1} N_{r}$ and

$$
\left(\begin{array}{cc}
X_{r} & -Y_{r} \\
-N_{r} & D_{r}
\end{array}\right)\left(\begin{array}{cc}
D_{l} & Y_{l} \\
N_{l} & X_{l}
\end{array}\right)=I
$$

represent a DCF of $F$ where the factors are given as in [7]. Then, the following represent a DCF of $f$ :

$$
\begin{gathered}
f=n_{l} d_{l}^{-1}=d_{r}^{-1} n_{r} \\
\left(\begin{array}{cc}
x_{r} & -y_{r} \\
-n_{r} & d_{r}
\end{array}\right)\left(\begin{array}{ll}
d_{l} & y_{l} \\
n_{l} & x_{l}
\end{array}\right)=I
\end{gathered}
$$

where $n_{l}=L^{-1}\left(N_{l}\right), d_{l}=L^{-1}\left(D_{l}\right), x_{l}=L^{-1}\left(X_{l}\right), y_{l}=$ $L^{-1}\left(Y_{l}\right), n_{r}=L^{-1}\left(N_{r}\right), \quad d_{r}=L^{-1}\left(D_{r}\right), \quad x_{r}=L^{-1}\left(X_{r}\right)$, $y_{r}=L^{-1}\left(Y_{r}\right)$ are in $\mathscr{L}_{T V}$ and $N$-periodic. Moreover, all stabilizing time-varying controllers $c$ of $f$ are given by

$c=\left(y_{l}-d_{l} q\right)\left(x_{l}-n_{l} q\right)^{-1}=\left(x_{r}-q n_{r}\right)^{-1}\left(y_{r}-q d_{r}\right)$

where $q \in \mathscr{L}_{T V}$.

Note that, in the above fact, it is easy to check that $q$ is $N$-periodic if and only if $c$ is $N$ periodic. Finally, along the lines of [16], it is shown in [1] that the optimal performance in periodic plants is achieved with periodic controllers, namely:

Fact 2.5: Let $H_{p}, U_{p}, V_{p}$ be $N$-periodic and stable causal linear operators. Then

$$
\inf _{Q \in \mathscr{L}_{T V}}\left\|H_{p}-U_{p} Q V_{p}\right\|=\inf _{Q_{p}}\left\|H_{p}-U_{p} Q_{p} V_{p}\right\|
$$

where $Q_{p}$ is $N$-periodic and in $\mathscr{L}_{T V}$.

\section{Problem Definttron}

The standard block diagram for the disturbance rejection problem is depicted in Fig. 1 . In this figure, $P_{p}$ denotes some fixed linear causal $N$-periodic plant, $C_{v}$ denotes a time-varying compensator (not necessarily periodic), and the signals $w, z, y$, and $u$ are defined as follows: $w$, exogenous disturbance; $z$, signals to be regulated; $y$, measured plant output; and $u$, control inputs to the plant. $P_{p}$ can be thought of as a four block matrix each block being a linear causal $N$-periodic system. The following common assumptions are made.

Assumption 3.1: The system of Figure 1 is well-posed [5], [7].

Assumption 3.2: $P_{p}$ is finite-dimensional and stabilizable.

A sufficient condition for Assumption 3.1 to hold is that the lower diagonal block of $P_{p}$ is strictly causal. Let $T_{z w}$ represent the resulting map from $w$ to $z$ for a given compensator $C_{v}$. Our objective can be now stated as OBJ:

find $C_{v}$ such that the resulting closed-loop system is stable and also the induced norm $\left\|T_{z w}\right\|$ over $l^{\infty}$ is minimized.

In the sequel, we show that this problem can be turned into an equivalent LTI problem. Towards this end, we lift each of the four blocks of $P_{p}$ and let $P$ denote the resulting LTI plant. Consider now, the disturbance rejection problem for

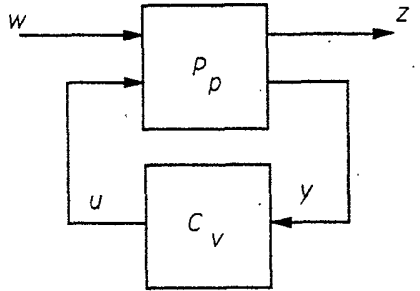

Fig. 1. Block diagram of disturbance rejection.

the system $P$ and let $\tilde{T}_{z w}$ denote the map from $\tilde{w}=W w$ to $\tilde{z}=W z$. Note that the Assumption 3.1 guarantees the wellposedness of the LTI problem. Then it is well known [18], [6], [17], [5] that all the feasible maps are given as $\tilde{T}_{z w}=H$ - UQV where $H, U, V \in \mathscr{L}_{T I}$ and $Q \in \mathscr{L}_{T V}$. Moreover, $H, U, V$ are determined by $P$. Now, the following lemma shows the aforementioned equivalent.

Lemma 3.1: The OBJ is equivalent to the problem:

$$
\inf _{Q \in \mathscr{L}_{T I}}\|H-U Q V\|
$$

subject to $Q(0)$ being block lower triangular.

Proof: From Fact 2.4 and Fact 2.5 we obtain

$$
\inf _{C_{v}}\left\|T_{z w}\right\|=\inf _{Q_{p}}\left\|H_{p}-U_{p} Q_{p} V_{p}\right\|
$$

where $Q_{p}$ is $N$-periodic, stable, and $H_{p}=L^{-1}(H), U_{p}=$ $L^{-1}(U), V_{p}=L^{-1}(V)$. Now, by Facts 2.2 and 2.3 it follows that

$$
\inf _{Q_{p}}\left\|H_{p}-U_{p} Q_{p} V_{p}\right\|=\inf _{Q=L\left(Q_{p}\right)}\|H-U Q V\| .
$$

It is problem OPT that we approach in the following section.

\section{PROBLEM SOLUTION}

Clearly, if in OPT we remove the constraint on $Q(0)$ then the problem becomes the standard $l^{1}$ optimization problem [2], [3]. In [2], [3] the authors solve the problem by solving the dual problem with linear programming methods. We can solve OPT by extending the method in [2], [3] to account for the constraint on $Q(0)$. To show this, assume that $\hat{U}(\lambda), \hat{V}(\lambda)$ have full-row and column rank, respectively, for almost all $\lambda$; we will come back to the general case later on. Also, assume that $\hat{U}(\lambda), \hat{V}(\lambda)$ have no zeros on the unit circle. Let now $\left\{P_{n}\right\}_{n=1}^{N_{s}}$ be as in [2], [3] the basis for the functionals in $c_{m \times n}^{0}$ that annihilate the space

$$
S_{s}=\left\{K: K=U Q V, Q \in l_{m \times n}^{1}\right\},
$$

i.e.,

$$
\left\langle U Q V, P_{i}\right\rangle=0 \quad \forall i=1,2, \cdots, N_{s}, \quad, Q \in l_{m \times n}^{1} .
$$

These functionals are attributed to the unstable zeros of $U(\lambda)$ and $V(\lambda)$. Suppose now that we are able to find functionals $\left\{X_{j}\right\}_{j=1}^{J}$ in $c_{m \times n}^{0}$ having the following property:

if $K \in S_{s}$ then

$$
\left\langle K, X_{j}\right\rangle=0 \quad \forall_{j}=1,2, \cdots, J
$$


if and only if

$\exists Q \in l_{m \times n}^{1}$ with $K=U Q V$ and

$$
Q(0) \text { block lower triangular. }
$$

Next, define $S$ as

$$
S=\left\{K: K=U Q V, Q \in l_{m \times n}^{1}, Q(0)\right.
$$

block lower triangular $\}$.

The following lemma, given without proof, stems from standard results in functional analysis (for example, [10]).

Lemma 4.1: Let $\left\{P_{n}\right\}_{n=1}^{N_{s}} \in c_{m \times n}^{0}$ as above and let $\left\{X_{j}\right\}_{j=1}^{J}$ in $c_{m \times n}^{0}$ satisfy PROP as above. Then the annihilator subspace ${ }^{\perp} S$ of $S$ can be characterized as

$$
{ }^{\perp} S=\operatorname{span}\left(\left\{P_{n}\right\}_{n=1}^{N_{s}} \cup\left\{X_{j}\right\}_{j=1}^{J}\right) .
$$

All that the above lemma says is that the functionals $\left\{X_{j}\right\}_{j=1}^{J}$ add the extra constraints of causality of $Q(0)$ required to solve OPT by enlarging the subspace ${ }^{\perp} S_{s}$ to ${ }^{\perp} S$. Since we now have a complete characterization of ${ }^{\perp} S$ we can proceed exactly as in [2], [3] to solve OPT. Namely, using duality we can transform OPT to a maximization problem inside $B\left({ }^{\perp} S\right)$.

By Fact $2.1\left(c_{m \times n}^{0}\right)^{*}=l_{m \times n}^{1}$; moreover, if $M$ is the subspace in $c_{m \times n}^{0}$ defined as $M=\operatorname{span}\left(\left\{P_{n}\right\}_{n=1}^{N_{s}} U\right.$ $\left\{X_{j}\right\}_{j=1}^{J}$ ) then from the definitions of $\left\{X_{j}\right\}_{j=1}^{J}$ and $\left\{P_{n}\right\}_{n=1}^{N_{s}}$ it is easy to verify as in [2], [3] that $M^{\perp}=S$ which implies that $S$ is weak * closed. Hence

$$
\inf _{K \in S}\|H-K\|=\min _{K \in\left({ }^{\perp} S\right)^{\perp}}\|H-K\|=\sup _{G \in B\left({ }^{\perp} S\right)}\langle G, H\rangle
$$

but since ${ }^{\perp} S$ is finite-dimensional

$$
\sup _{G \in B\left({ }^{\perp} S\right)}\langle G, H\rangle=\max _{G \in B\left({ }^{\perp} S\right)}\langle G, H\rangle
$$

therefore

$$
\inf _{K \in S}\|H-K\|=\max _{G \in B\left({ }^{\perp} S\right)}\langle G, H\rangle .
$$

The right-hand side of the above equality can be turned into a finite-dimensional linear programming problem [2], [3] and hence we obtain the optimal $G=G_{0}$. The optimal $K_{0}$ is found by using the alignment conditions [2], [3]

$$
\left\langle G_{0}, H-K_{0}\right\rangle=\left\|H-K_{0}\right\| .
$$

In the sequel we show how to obtain these $\left\{X_{j}\right\}_{j=1}^{J}$.

First, we associate a functional $R_{j}=\left\{R_{j}(0), 0,0, \cdots,\right\}$ in $c^{0}$ for every element $j$ of $Q(0)$ with indexes $\left(l_{j}, m_{j}\right)$ that has to equal 0 (i.e., the elements that are not in the block lower triangular portion of $Q(0)$ ) as follows: we pick $R_{j}(0)$ to have the same dimension as $Q(0)$ and also to have all its entries but one equal to 0 . The nonzero entry is taken to equal 1 and its indexes are precisely the ones that correspond to $j$ (i.e., $\left(l_{j}, m_{j}\right)$ ). Hence, if $r$ equals the number of elements in $Q(0)$ that are necessarily zero then

$$
\left\langle Q, R_{j}\right\rangle=0 \quad \forall j=1,2, \cdots, r
$$

if and only if

$$
Q(0) \quad \text { block lower triangular. }
$$

Therefore OPT can be rewritten as

$$
\underset{Q}{\inf \|H-U Q V\|}
$$

with

$$
Q \in l^{1},\left\langle Q, R_{j}\right\rangle=0 \quad \forall j=1,2, \cdots, r .
$$

Next, we present the solution to OPT by following two approaches.

Approach 1: By performing an inner-outer factorization [7] for $U, V$ we obtain

$$
U=U_{i} U_{o}, \quad V=V_{o} V_{i}
$$

where the subscript $i$ stands for "inner" and $o$ for "outer," i.e., $\hat{U}_{i}^{T}\left(\lambda^{-1}\right) \hat{U}_{i}(\lambda)=I$ and $\hat{V}_{i}\left(\lambda^{-1}\right) \hat{V}_{i}^{T}(\lambda)=I$. Note that $\hat{U}_{i}(\lambda), \hat{V}_{i}(\lambda)$ are square matrices. Also, the various factors $U_{i}, U_{o}, V_{o}, V_{i}$ may not, in general, correspond to an $N$-periodic system (i.e., at $\lambda=0$ they may not possess the block lower triangular structure). Our goal is to reflect the constraint on $Q(0)$ to the product $K=U Q V$. Note that if $U(0)$ and $V(0)$ are square and invertible matrices then the situation is simple. To realize this, note that by Fact 2.4 both $U(0), V(0)$ are block lower triangular. Hence, since $K(0)=$ $U(0) Q(0) V(0)$, we have that $Q(0)$ is block lower triangular if and only if $K(0)$ is block lower triangular. Therefore, the functionals we are looking for are simply the $R_{j}$ 's as defined in the beginning of the section. However, this might not be the case: $U(0), V(0)$ might not be square and/or they might not be invertible due to, for example, a delay. Because of this, we proceed by reflecting the constraints first on $U_{o} Q V_{o}$ and then on $K$. Towards this end let $Z=U_{o} Q V_{o}$; the following propositions show how $Z$ is affected due to the constraints on $Q$.

Proposition 4.1: Let $Z \in l^{1}$ then

$\exists Q \in l^{1}$ with $\left\langle Q, R_{j}\right\rangle=0$

$$
\forall j=1,2, \cdots, r \text { and } Z=U_{o} Q V_{o}
$$

if and only if

$Z(0) \in S_{A}=\left\{U_{o}(0) A V_{o}(0)\right.$

\section{$A$ block lower triangular matrix $\}$.}

Proof: The "if" direction goes as follows: let $U_{o r}, V_{o l}$ denote any right and left stable inverses of $U_{o}, V_{o}$, respectively. Then $U_{o r}, V_{o l} \in l^{1}$. Let $A$ be a block lower triangular matrix such that $Z(0)=U_{o}(0) A V_{o}(0)$; define $Q_{A}=$ $\{A, 0,0, \cdots$,$\} and let \tilde{Z}=Z-U_{o} Q_{A} V_{o}$ then $\tilde{Z} \in l^{1}$ and $\tilde{Z}(0)=0$. Define now $Q$ as $Q=U_{o r} \tilde{Z} V_{o l}+Q_{A}$. It then follows that $Q \in l^{1}, Q(0)$ is block lower triangular and $Z=$ $U_{o} Q V_{o}$..

The "only if" direction is immediate.

Proposition 4.1 shows that only $Z(0)$ is constrained to lie in a certain subspace (i.e., $S_{A}$ ) otherwise $Z$ can be arbitrary in $l^{1}$. Hence, the problem of specifying constraints on $Z$ in terms of functionals is finite-dimensional. Note that the characterization of this subspace is independent of the choice of right and left inverses for $U_{o}, V_{o}$, respectively, hence it is exact. In the next proposition the annihilator subspace $S_{B}$ of 
$S_{A}$ is characterized and from this the annihilator subspace of $Z$ is generated

Proposition 4.2: Let $\left\{B_{j}\right\}_{j=1}^{j B}$ be a basis for the finitedimensional Euclidean space

$$
S_{B}=\left\{B: U_{o}^{T}(0) B V_{o}^{T}(0) \in \operatorname{span}\left(\left\{R_{j}(0)\right\}_{j=1}^{r}\right)\right\} .
$$

Define the following functionals in $c^{0}$ :

$$
R_{z_{j}}=\left\{B_{j}, 0,0, \cdots,\right\} \quad \forall j=1,2, \cdots, j_{B} .
$$

Let $Z \in l^{1}$ then

$$
Z(0) \in S_{A}
$$

if and only if

$$
\left\langle Z, R_{z_{j}}\right\rangle=0 \quad \forall j=1,2, \cdots, j_{B} .
$$

Proof: Since the only nonzero element in $R_{z_{j}}$ is the first one, it is clear that the condition $\left\langle Z, R_{z_{j}}\right\rangle=0$ is equivalent to the condition $\left\langle Z(0), B_{j}\right\rangle=0$ where $\langle\cdot, \cdot\rangle$ is interpreted as an inner product in the finite-dimensional Euclidean space of matrices. Now, let $A$ be a block lower triangular matrix and $B \in S_{A}^{\perp}$; then since

$$
\left\langle U_{o}(0) A V_{o}(0), B\right\rangle=\left\langle A, U_{o}^{T}(0) B V_{o}^{T}(0)\right\rangle \quad \forall A
$$

it follows that $B \in S_{B}$. Conversely, it also follows that if $B \in S_{B}$ then $\left\langle U_{o}(0) A V_{o}(0), B\right\rangle=0$. Hence

$$
S_{A}^{\perp}=S_{B}
$$

or equivalently

$$
S_{B}^{\perp}=S_{A}
$$

which proves the proposition.

The computation of $\left\{B_{j}\right\}_{j=1}^{j_{B}}$ for the general nonsquare case is routine and is presented in the Appendix. Since the matrices $U_{o}(0), V_{o}(0)$ have full-row and column rank, respectively, it can be easily checked that the dimension of $S_{B}$ is going to be at most equal to the dimension of $\operatorname{span}\left(\left\{R_{j}(0)\right\}_{j=1}^{r}\right)$, i.e., $j_{B} \leq r$. Therefore, $Z$ will be "less constrained" than $Q$. In fact, there are cases as in Example 2 , where $Z$ turns out to be unconstrained in $l^{1}$. In this case, $S_{B}=\{0\}$ or equivalently $S_{A}$ is the whole finite-dimensional space of matrices (of appropriate dimensions) and hence, the solution to OPT is the same to the standard unconstrained problem in [2], [3]. The equality $j_{B}=r$ occurs when $U$ and $V$ are square. In this case, the computation of $\left\{B_{j}\right\}_{j=1}^{j_{B}}$ is immediate. Namely

$$
B_{j}=U_{o}^{-T}(0) R_{j}(0) V_{o}^{-T}(0) \quad j=1, \cdots, r .
$$

In view of the above propositions, OPT can be stated as

$$
\inf _{Z}\left\|H-U_{i} Z V_{i}\right\|
$$

with

$$
Z \in l^{1},\left\langle Z, R_{z_{j}}\right\rangle=0 \quad \forall j=1, \cdots, j_{B} .
$$

We now show how to obtain the functionals $\left\{X_{j}\right\}_{j=1}^{J}$ that have the property (PROP) mentioned in the beginning of this section.
Theorem 4.1: The functionals

$$
X_{j}=U_{i} R_{z_{j}} V_{i} \quad j=1,2, \cdots, j_{B}
$$

satisfy PROP.

Proof: Consider the bounded operators $T_{U_{i}}, T_{V_{i}}$ on $l^{1}$ defined as

$$
\begin{aligned}
& \left(T_{U_{i}} X\right)(t)=\sum_{\tau=0}^{t} U_{i}(\tau) X(t-\tau) \\
& \left(T_{V_{i}} X\right)(t)=\sum_{\tau=0}^{t} X(\tau) V_{i}(t-\tau)
\end{aligned}
$$

where $X \in l^{1}$. Their weak ${ }^{*}$ adjoints $T_{U_{i}}^{*}, T_{V_{i}}^{*}$ on $c^{0}$ which are given by

$$
\begin{aligned}
& \left(T_{U_{i}}^{*} Y\right)(t)=\sum_{\tau=0}^{\infty} U_{i}^{T}(\tau) Y(\tau+t) \\
& \left(T_{V_{i}}^{*} Y\right)(t)=\sum_{\tau=0}^{\infty} Y(\tau+t) V_{i}^{T}(\tau)
\end{aligned}
$$

where $Y \in c^{0}$.

Notice, that since $U_{i}, V_{i}$ inner then $\hat{U}_{i}^{T}\left(\lambda^{-1}\right) \hat{U}_{i}(\lambda)=I$ and $\hat{V}_{i}\left(\lambda^{-1}\right) \hat{V}_{i}^{T}(\lambda)=I$. Note also that $T_{U_{i}}^{*}, T_{U_{i}}$ represent multiplication from the right whereas $T_{V_{i}}^{*}, T_{V_{i}}$ represent multiplication from the left. Hence, it follows that $T_{U_{i}}^{*} T_{U_{i}}=I$ and $T_{V_{i}}^{*} T_{V_{i}}=I$.

Interpreting

and

$$
U_{i} Z V_{i}=T_{U_{i}}\left(T_{V_{i}}(Z)\right)
$$

with

$$
U_{i} R_{z} V_{i}=T_{U_{i}}\left(T_{V_{i}}\left(R_{z}\right)\right)
$$

we can verify that

$$
R_{z} \in \operatorname{span}\left(\left\{R_{z_{j}}\right\}_{j=1}^{j_{B}}\right)
$$

$$
\left\langle U_{i} Z V_{i}, U_{i} R_{z} V_{i}\right\rangle=\left\langle Z, R_{z}\right\rangle .
$$

Hence, if $X=U_{i} R_{z} V_{i}$ then

$$
\langle U Q V, X\rangle=0 \quad \text { if and only if }\left\langle Z, R_{z}\right\rangle=0
$$

which completes the proof.

Remark: So far in this section we assumed that $\hat{U}(\lambda), \hat{V}(\lambda)$ have full-row and column rank, respectively. However, there is no loss of generality since in the "bad" rank case (i.e., when the above assumption does not hold [2], [3], [11]) it is shown in [11] that in order to solve the unconstrained problem it is necessary to solve a square subproblem. In particular, we can partition $U, V$ as

$$
U=\left(\begin{array}{c}
\bar{U} \\
U_{2}
\end{array}\right), \quad V=\left(\begin{array}{ll}
\bar{V} & V_{2}
\end{array}\right)
$$

where $\bar{U}, \bar{V}$ are square and invertible. Let $K=U Q V$ then

$$
K=\left(\begin{array}{cc}
\bar{K} & K_{12} \\
K_{21} & K_{22}
\end{array}\right) .
$$

A necessary condition for the existence of a solution is that $\bar{K}$ interpolates $\bar{U}, \bar{V}$ which is the aforementioned subproblem. 
The solution presented so far required an inner-outer factorization for $U, V$. Next, an alternative approach which does not require inner-outer factorization is presented. For simplicity, we indicate the method in the case where $V=I$. The extension in the case where $V \neq I$ should be immediate to the reader.

Approach 2: Let $U$ be written in the Smith form [8] as

$$
U=S_{1} \Sigma\left(\begin{array}{ll}
I & 0
\end{array}\right) S_{2}
$$

where $S_{1}, S_{2}$ correspond to square, stable transfer matrices with stable inverses and

$$
\hat{\Sigma}(\lambda)=\operatorname{diag}\left(\hat{G}_{0}(\lambda), \lambda \hat{g}_{1}(\lambda), \cdots, \lambda^{k} \hat{g}_{k}(\lambda)\right)
$$

where $\hat{G}_{0}(\lambda)$ is a diagonal stable transfer matrix with no zeros at $\lambda=0$, and $\hat{g}_{1}(\lambda), \cdots, \hat{g}_{k}(\lambda)$ are scalar stable transfer functions also with no zeros at $\lambda=0$. Let $\left\{\bar{B}_{j}\right\}_{j=1}^{j \bar{B}_{B}}$ be a basis for the finite-dimensional space

$$
S_{\bar{B}}=\left\{\bar{B}: S_{2}^{T}(0)\left(\begin{array}{l}
I \\
0
\end{array}\right) \bar{B} \in \operatorname{span}\left(\left\{R_{j}(0)\right\}_{j=1}^{r}\right)\right\} .
$$

Theorem 4.2: Let $T_{S_{1}^{-1}}^{*}$ be the weak ${ }^{*}$ adjoint operator on $c^{0}$ associated with $S_{1}^{-1}$, i.e.,

$$
\left(T_{S_{1}^{-1}}^{*} Y\right)(t)=\sum_{\tau=0}^{\infty} S_{1}^{-T}(\tau) Y(\tau+t)
$$

with $Y \in c^{0}$. Then the functionals

$$
X_{j}=T_{S_{1}^{-1}}^{*}\left(D_{j}\right)
$$

with

$$
\hat{D}_{j}(\lambda)=\operatorname{diag}\left(\hat{G}_{0}^{-T}(0), \lambda \hat{g}_{1}^{-1}(0), \cdots, \lambda^{k} \hat{g}_{k}^{-1}(0)\right) \hat{F}_{j}(\lambda)
$$

with

$$
F_{j}=\left\{\bar{B}_{j}, 0,0, \cdots,\right\}
$$

for $j=1,2, \cdots, j_{\bar{B}}$ satisfy PROP.

Proof: First, note that the term $(I 0) S_{2}$ is "outer" and $S_{2}^{-1}\left(\begin{array}{l}I \\ 0\end{array}\right)$ is a stable right inverse of it. Hence, if $\bar{Q}=(I$ 0) $S_{2} Q$ then we can go through exactly the same arguments as in Propositions 4.1 and 4.2 to show that if $\bar{Q} \in l^{1}$ then

$\exists Q \in l^{1}$ with $\left\langle Q, R_{j}\right\rangle=0$

$$
\forall j=1,2, \cdots, r \text { and } \bar{Q}=\left(\begin{array}{ll}
I & 0
\end{array}\right) S_{2} Q
$$

if and only if

$$
\left\langle\bar{Q}, F_{j}\right\rangle=0 \quad \forall j=1,2, \cdots, j_{\bar{B}} .
$$

Now, let $F \in \operatorname{span}\left(\left\{F_{j}\right\}_{j=1}^{j_{\tilde{B}}}\right)$ and define $D \in c^{0}$ as

$$
\hat{D}(\lambda)=\operatorname{diag}\left(\hat{G}_{0}^{-T}(0), \lambda \hat{g}_{1}^{-1}(0), \cdots, \lambda^{k} \hat{g}_{k}^{-1}(0)\right) \hat{F}(\lambda)
$$

then we can verify that

$$
\langle\Sigma \bar{Q}, D\rangle=\langle\bar{Q}, F\rangle
$$

as follows.

$$
\begin{gathered}
\text { Partition } \bar{Q} \text { and } F \text { as } \bar{Q}=\left(\begin{array}{c}
Q_{0} \\
q_{1} \\
\vdots \\
q_{k}
\end{array}\right) \text { and } F=\left(\begin{array}{c}
F_{0} \\
f_{1} \\
\vdots \\
f_{k}
\end{array}\right) \text { then } \\
\langle\Sigma \bar{Q}, D\rangle=\left\langle\hat{G}_{0}(\lambda) \hat{Q}_{0}(\lambda), \hat{G}_{0}^{-T}(0) \hat{F}_{0}(\lambda)\right\rangle \\
+\sum_{i=1}^{i=k}\left\langle\lambda^{i} \hat{g}_{i}(\lambda) \hat{q}_{i}(\lambda), \lambda^{i} \hat{g}_{i}^{-1}(0) \hat{f}_{i}(\lambda)\right\rangle
\end{gathered}
$$

but $\hat{F}_{0}(\lambda)=F_{0}(0)$ and $\hat{f}_{i}(\lambda)=f_{i}(0) \forall i=1,2, \cdots, k$ hence

$$
\begin{aligned}
\langle\Sigma \bar{Q}, D\rangle=\left\langle G_{0}(0) Q_{0}(0), G_{0}^{-T}(0) F_{0}(0)\right\rangle & \\
& +\sum_{i=1}^{i=k}\left\langle g_{i}(0) q_{i}(0), g_{i}^{-1}(0) f_{i}(0)\right\rangle=\langle\bar{Q}, F\rangle .
\end{aligned}
$$

As in the proof of Theorem 4.1, let $T_{S_{1}^{-3}}$ be the operator on $l^{1}$ associated with the stable system $S_{1}^{-1}$ and let $T_{S_{1}^{-1}}^{*}$ be its adjoint on $c^{0}$. Then by defining

we can show that

$$
X=T_{S_{1}^{-1}}^{*}(D)
$$

$$
\langle\Sigma \bar{Q}, D\rangle=\left\langle S_{1} \Sigma \bar{Q}, X\right\rangle
$$

Hence

$$
\left\langle S_{1} \Sigma \bar{Q}, X\right\rangle=0 \quad \text { if and only if }\langle\bar{Q}, F\rangle=0
$$

which completes the proof.

The functionals $X_{j}, j=1, \cdots, j_{\bar{B}}$ obtained by the second method will contain finitely many nonzero components. Namely, $X_{j}(m)=0 \forall m>k$. This is so because $F_{j}$ has all its elemenis but the first equal to zero and hence $D_{j}(m)$ equals zero for $m>k$. In the first method however, the obtained functionals will not, in general, have this property. They will decay though, (since they lie in $c^{0}$ ) and the rate of decay will be dictated by the poles of $U_{i}$ and $\dot{V}_{i}$. Hence, it seems that the linear programming problem in the dual space [2] will be simpler when the second method is used, provided that a simple way to obtain a Smith decomposition of a transfer matrix exists.

Next, we show that in general OPT has different solution than the unconstrained problem.

Example 1: Consider an optimization problem in a 2-periodic single-input single-output system with the equivalent LTI problem being as follows:

$$
\inf _{Q \in l^{1}}\|H-U Q\|
$$

such that $Q(0)$ is lower triangular, where $\hat{H}(\lambda)=\left(\begin{array}{ll}1 & 0 \\ 0 & 2\end{array}\right)$, $\hat{U}(\lambda)=\left(\begin{array}{cc}\lambda & -\lambda \\ 0 & 1\end{array}\right)$

We first solve the unconstrained problem following [2].

The basis for the functionals that annihilate $S_{s}=\{U Q: Q$ $\left.\in l^{1}\right\}$ consists of the following:

$$
\begin{aligned}
& F_{1}=\left\{\left(\begin{array}{ll}
1 & 0 \\
0 & 0
\end{array}\right), 0, \cdots,\right\} \\
& F_{2}=\left\{\left(\begin{array}{ll}
0 & 1 \\
0 & 0
\end{array}\right), 0, \cdots,\right\}
\end{aligned}
$$


The resulting optimal solution $\Phi_{u}=H-U Q_{u}$ is

$$
\Phi_{u}=\left(\begin{array}{cc}
1 & 0 \\
\phi_{12} & \phi_{22}
\end{array}\right)
$$

where $\phi_{12}, \phi_{22}$ arbitrary in $l^{1}$ such that

$$
\left\|\phi_{12}\right\|_{l^{1}}+\left\|\phi_{22}\right\|_{l^{1}} \leq 1
$$

and

$$
Q_{u}=\left(\begin{array}{ll}
-\phi_{21} & 2-\phi_{22} \\
-\phi_{21} & 2-\phi_{22}
\end{array}\right)
$$

Also,

$$
\left\|\Phi_{u}\right\|_{l^{1}}=1
$$

Now, in the constrained case, we obtain by using the second approach the following extra functional:

$$
F_{3}=\left\{\left(\begin{array}{ll}
0 & 0 \\
0 & 1
\end{array}\right),\left(\begin{array}{ll}
0 & 1 \\
0 & 0
\end{array}\right), 0, \cdots,\right\} .
$$

The resulting optimal solution is:

with

$$
\hat{\Phi}(\lambda)=\left(\begin{array}{cc}
1 & 0.5 \lambda \\
0 & 1.5
\end{array}\right)
$$

$$
\hat{Q}(\lambda)=\left(\begin{array}{ll}
0 & 0 \\
0 & 0.5
\end{array}\right)
$$

Also,

$$
\|\Phi\|_{\gamma^{1}}=1.5 \text {. }
$$

First, note that the optimal $Q$ obtained for the constrained case does have the lower triangular structure at $\lambda=0$. Also notice that the optimal performance is (as expected) worse in the constrained case than in the unconstrained one. Moreover, we demonstrate that if we just "project" $Q_{u}$ so that it corresponds to a causal periodic controller we do not necessarily obtain optimal performance.

Let $Q_{u p}$ denote the causal projection of $Q_{u}$, i.e.,

$$
Q_{u p}=\left(\begin{array}{cc}
-\phi_{21} & -\phi_{22}+\phi_{22}(0) \\
-\phi_{21} & 2-\phi_{22}
\end{array}\right)
$$

and let $\Phi_{u p}=H-U Q_{u p}$ then

$$
\hat{\Phi}_{u p}(\lambda)=\left(\begin{array}{cc}
1 & \lambda\left(2-\phi_{22}(0)\right) \\
-\phi_{21} & -\phi_{22}
\end{array}\right) .
$$

Hence, since $\left\|\phi_{21}\right\|_{l^{1}}+\left\|\phi_{22}\right\|_{l^{1}} \leq 1$ we have that

$$
\left\|\Phi_{u p}\right\|_{l^{1}} \geq 2
$$

thus $\left\|\Phi_{u p}\right\|_{l^{1}}>\|\Phi\|_{l^{1}}$.

There are cases, however, where the unconstrained problem and the OPT are the same. This is indicated in the following example.

Example 2: Once again consider an optimization problem in a 2-periodic system with one input and one output and let the equivalent LTI problem be given as

$$
\inf _{Q \in l^{1}}\|H-U Q\|
$$

where $H$ is $2 \times 2, U$ is $2 \times 4, Q$ is $4 \times 2$ with $Q(0)$ block lower triangular. Suppose $U=U_{i} U_{o}$ with $\hat{U}_{i}(\lambda)=\lambda, \hat{U}_{o}(\lambda)$ $=\left(\begin{array}{ll}0 & I\end{array}\right)$ then $U_{o}(0)$ has full-row rank. Finally, let $Z=U_{o} Q$. It can be easily checked that $U_{o}(0) Q(0)$ spans the whole $2 \times 2$-dimensional space of real matrices with $Q(0)$ being block lower triangular, i.e., $Q(0)$ of the form

$$
Q(0)=\left(\begin{array}{ll}
* & 0 \\
* & 0 \\
* & * \\
* & *
\end{array}\right) .
$$

Hence, the problem is

$$
\inf _{Z \in l^{1}}\left\|H-U_{i} Z\right\|
$$

which is exactly the unconstrained problem.

\section{Applications}

\section{A. $l^{\infty}$ Optimal Disturbance Rejection in Multirate Sampled Data Systems}

An important feature of the solution presented in this paper is its immediate applicability to the problem of optimal disturbance rejection in multirate sampled data (MRSD) systems. The most general $m$-input $p$-output MRSD system is shown in Fig. 2. This system consists of a LTI continuous plant $G_{c}$ together with multirate hold and sampling mechanisms which are dictated by the sets of positive integers $\left\{L_{i}\right\}_{i=1}^{p},\left\{K_{j}\right\}_{j=1}^{m}$ and the shortest time period $T$ as follows: each value of the input sequence $\left\{u_{j}(k)\right\}_{k=0}^{\infty}$ with $j=$ $1, \cdots, m$ is held for time $K_{j} T$ and each value of the output sequence $\left\{y_{i}(k)\right\}_{k=0}^{\infty}$ with $i=1, \cdots, p$ is obtained by sampling the output of the continuous plant at multiples of $L_{i} T$.

As it was shown in [12] MRSD systems belong in a more general class of time varying systems, i.e., the so-called $\left(P_{i}, M_{j}\right)$ shift-varying systems. These are $m$-input $p$-output discrete systems associated with sets of positive integers $\left\{P_{i}\right\}_{i=1}^{p},\left\{M_{j}\right\}_{j=1}^{m}$ and an input-output map $f$ that has the following property: if each input sequence $u_{j}$ is shifted by $M_{j}$ then each output sequence $y_{i}$ will be shifted by $P_{i}$. In particular, for the system in Fig. 2 if the integers $\left(L_{1}\right.$, $\left.L_{2}, \cdots, L_{p}, K_{1}, K_{2}, \cdots, K_{m}\right)$ are relative prime and if $N$ denotes their least common multiple then it corresponds to a $\left(P_{i}, M_{j}\right)$ shift-varying system with $P_{i}=\left(N / L_{i}\right)$ and $M_{j}=$ $\left(N / K_{j}\right)$. Note that $N$-periodic systems belong in the class of $\left(P_{i}, M_{j}\right)$ shift-varying systems with $P_{i}=M_{j}=N \forall i, j$. In [12], [15], a lifting technique was used to show that these systems are equivalent in the strong sense (just as in the case of $N$-periodic) to a class of $M$-input $P$-output LTI systems, where $P=\sum_{i=1}^{p} P_{i}$ and $M=\sum_{j=1}^{m} M_{j}$. The $\lambda$-transform of this class of LTI systems should satisfy constraints at $\lambda=0$ analogous to the $N$-periodic case. These constraints are referred to in [13] as $\left(P_{i}, M_{j}\right)$ causality conditions. More specifically, let $\hat{G}(\lambda)$ represent the equivalent LTI system of a $\left(P_{i}, M_{j}\right)$ shift-varying system (for example the MRSD in Fig. 2) and let $D=\hat{G}(0)$. Partition the matrix $D$ as follows:

$$
D=\left(\begin{array}{cccc}
D_{11} & D_{12} & \cdots & D_{1 m} \\
\cdot & \cdot & \cdot & \cdot \\
D_{p 1} & D_{p 2} & \cdots & D_{p m}
\end{array}\right)
$$




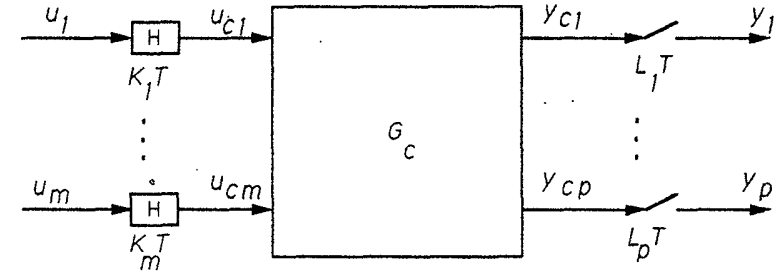

Fig. 2. General multirate sampled data system.

where each $D_{i j}$ is a $P_{i} \times M_{j}$ matrix. The $\left(P_{i}, M_{j}\right)$ causality conditions are the following [13]:

$$
\begin{gathered}
\left(D_{i j}\right)_{\alpha \beta}=0 \\
\text { when }(\alpha-1) \frac{N}{P_{i}}-(\beta-1) \frac{N}{M_{j}}<0 ; \\
1 \leq \alpha \leq P_{i}, 1 \leq \beta \leq M_{j} .
\end{gathered}
$$

In [13], [15] a parametrization of all $\left(M_{j}, P_{i}\right)$ stabilizing controllers $C_{m r}$ was obtained by using the parametrization in [7] of all LTI stabilizing controllers of the lifted LTI system. The observation was (compare Fact 2.4) that the right and left Bezout elements of the lifted system satisfy the causality conditions. Hence, the so-obtained LTI controllers parametrized by the Youla free parameter $Q$, satisfy the $\left(M_{j}, P_{i}\right)$ causality conditions if and only if $Q$ satisfies the $\left(M_{j}, P_{i}\right)$ causality conditions. Moreover, these controllers can be realized as causal multirate controllers using the architectures of [13]. It should be emphasized [13], [15] that if $C_{m r}$ is a causal multirate controller it does not necessarily mean that it is an input-output map of a continuous MRSD system; MRSD systems are a subclass of multirate systems.

In view of all the above, the problem of optimal disturbance rejection in a MRSD system is transformed to the same problem in the lifted LTI system. Namely, let $w$ represent the exogenous disturbances entering the system, $z$ the regulated output, and let $T_{z w}$ be the map from $w$ to $z$. Then

$$
\inf _{C_{m r}}\left\|T_{z w}\right\|=\inf _{Q}\|H-U Q V\|
$$

where $H, U, V$ are (causal) stable LTI systems and $Q$ is a (causal) stable LTI system that satisfies the $\left(M_{j}, P_{i}\right)$ causality conditions. A word of caution should be stated here: We implicitly assumed that $w$ enters the system in a discrete fashion. This might not be the case since part of the disturbances might be of continuous nature entering the continuous part of the system. Also, the output of interest $z$ might not be a discrete signal. However, we model a continuous signal as an output of a discrete one via a sample-and-hold element of sufficiently small period. The optimal solution to the purely discrete problem can yield suboptimal solutions for the original hybrid system. The degree of accuracy will depend on the period of the sample-and-hold element that we use to approximate the continuous signal. With this in mind, we will consider the purely discrete time problem defined above.

This problem is not at all much different from what we encountered for the $N$-periodic systems. In fact, we can proceed exactly as in the $N$-periodic case by simply modifying the functionals $R_{i}$ defined in the previous section as follows: let $J$ be the number of elements in $Q(0)$ that are by the $\left(M_{j}, P_{i}\right)$ causality conditions necessarily zero. To each $j \in\{1,2, \cdots, J\}$ we associate the indexes of the element of $Q(0)$ that is necessarily zero. Also, for each $j \in\{1,2, \cdots, J\}$ consider a matrix $L_{j}$ with the same dimension as $Q(0)$ that has all its entries but one equal to 0 . The nonzero entry is taken to equal 1 and its indexes are taken to be the ones that correspond to $j$ in the $Q(0)$ matrix. Define the functionals $\left\{R_{j}\right\}_{j=1}^{J}$ as

$$
R_{j}(0)=L_{j} ; R_{j}(k)=0, \quad k=1,2 \cdots .
$$

It is now clear that the solution of the $N$-periodic case applies immediately. Thus, if we constrain ourselves to $\left(M_{j}, P_{i}\right)$ compensators to solve the optimal disturbance rejection problem, then exactly the same methods of the previous section apply. Moreover, the results in [1] can be extended to show that optimal performance in a $\left(P_{i}, M_{j}\right)$ system can be achieved by a $\left(M_{j}, P_{i}\right)$ compensator. Therefore, we can obtain a complete solution to the optimal disturbance rejection problem for MRSD systems.

\section{B. Robust Stabilization of Periodic and Multirate Systems}

In this subsection, we start by considering the problem of robust stabilization in periodic systems. In particular, we indicate that necessary and sufficient conditions for robust stability can be obtained by considering the same problem for the equivalent lifted system and using the results in [4], [14], [16]. More specifically, we encounter the situation depicted in Fig. 3: we are given a nominal $m$-input $n$-output $N$-periodic strictly causal plant $G_{p}$ subject to additive unstructured perturbations. The perturbed plant is described as $G_{p}+$ $R_{p} \Delta_{v}$, where $R_{p}$ is a known $m$-input $r$-output $N$-periodic causal time-varying system, and $\Delta_{v}$ an unknown $r$-input $n$-output perturbation in a class $\mathscr{D}$ defined as

$$
\mathscr{D}=\left\{\Delta_{v}: \Delta_{v} \in \mathscr{L}_{T V}^{r \times n} \text { strictly causal with }\left\|\Delta_{v}\right\|<1\right\} .
$$

The objective is to find necessary and sufficient conditions for the existence of a single causal compensator $C_{v}$ that stabilizes the system in Fig. 3 for all $\Delta_{v} \in \mathscr{D}$. Note that the strict causality conditions on $G_{p}, \Delta_{v}$ exist exclusively to guarantee well-posedness of the problem [5], [7]. Consider now the same problem for the lifted system and let $G, R, \Delta, C$ represent the lifts of $G_{p}, R_{p}, \Delta_{v}, C_{v}$, respectively (see Section II). Then, it is evident (see also Fact 2.3) that well-posedness as well as stability of the lifted system implies well-posedness as well as stability of the original system in Fig. 3 and vice versa. Hence, it is enough to deal with the lifted system. A sufficient condition for stability follows immediately from the application of the small gain theorem [5]. Namely, the system can be stabilized

$$
\inf \left\|C(I-G C)^{-1} R\right\| \leq 1 .
$$

In [4], [14], [16] it was shown that the above small gain condition is necessary as well as sufficient for stability when the perturbations are arbitrary time varying with norm bounded by 1 , i.e., these perturbations considered in [4], 


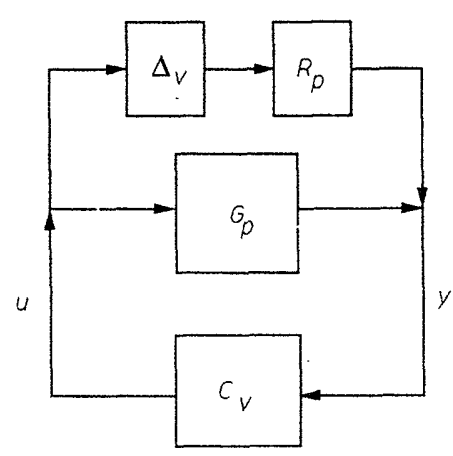

Fig. 3. Block diagram for robust stabilization.

[14], [16] are not initially constrained to be the image of a lifting isomorphism. However, as shown in [4], [14], [16] if the small gain condition does not hold then a strictly causal destabilizing $\Delta^{\circ} \in \mathscr{L}_{T V}^{r N \times n N}$ can be constructed such that the system becomes unstable for any controller. Now, the fact that $\Delta^{o}$ is strictly causal trivially implies that $\Delta^{o}$ is a legitimate perturbation for the lifted system, i.e., $\Delta^{\circ}$ corresponds to a $\Delta_{v}^{o}$ in $\mathscr{D}$. Therefore, the small gain condition is, in fact, necessary as well as sufficient for the stability of the original periodic system. Hence, if one wishes to design a stabilizing compensator $C_{v}$ for the periodic system that maximizes the tolerance to time-varying additive perturbations, the problem to solve is

$$
\inf _{C_{v} \text { stabilizing } G_{p}}\left\|C_{v}\left(I-G_{p} C_{v}\right)^{-1} R_{p}\right\|
$$

which can be transformed to

$$
\inf _{Q \in l^{1}}\|H-U Q V\|
$$

with $Q(0)$ block lower triangular and $H, U, V$ stable time invariant operators determined by $G, R$. It should be noted, that the situation does not change if we consider different types of perturbations (for example multiplicative): the small gain theorem gives necessary as well as sufficient conditions for stability.

Next, we encounter the problem of stability robustness in multirate systems. In particular, let $G_{m r}$ represent a $\left(P_{i}, M_{j}\right)$ shift-varying plant subject to additive unstructured multirate perturbations. Let the perturbed plant be $G_{m r}+R_{m r} \Delta_{m r}$ where $R_{m r}$ is a known $\left(N_{n}, M_{j}\right)$ shift-varying stable system and $\Delta_{m r}$ is an unknown $l^{\infty}$ stable causal multirate system with $\left\|\Delta_{m r}\right\|<1$. We stress that $\Delta_{m r}$ is not assumed to be a $\left(P_{n}, N_{n}\right)$ shift-varying system: it is viewed as an arbitrary stable causal multirate system in the context of [15]. The problem of robust stabilization is to find a single multirate controller $C_{m r}$ that stabilizes the perturbed class for all $\Delta_{m r}$. This problem can be treated analogously to the $N$-periodic case: let $G, R, C, \Delta$ be the lifts (using the lifting in [13], [15]) of $G_{m r}, R_{m r}, C_{m r}, \Delta_{m r}$, respectively. Note that $G, R$ are LTI and $\Delta$ is an arbitrary operator in $\mathscr{L}_{T V}^{P \times N}$ that satisfies the causality constraints of [15] with $\|\Delta\|<1$ where $N=\sum_{n} N_{n}, P=\sum_{i} P_{i}$. Again, using the results in [4], [14], [16] we can construct a strictly proper $\Delta^{o}$ with $\left\|\Delta^{o}\right\|<$ 1 for any $\left(M_{j}, P_{n}\right)$ causal controller that destabilizes the lifted system if and only if $\inf _{C}\left\|C(I-G C)^{-1} R\right\|>1$. This $\Delta^{\circ}$ trivially satisfies the causality conditions of [15] and hence it corresponds to a causal multirate $\Delta_{m r}^{o}$. Since stabil- ity of the lifted system implies stability of the original one and vice versa [13], [15], we conclude that the small gain condition is necessary as well as sufficient for the stability of the multirate system. Hence the optimal compensator $C_{m r}$ for robust stability to additive perturbations can be obtained by solving a performance problem in the lifted system. Namely

$$
\inf _{C \text { stabilizing } G}\left\|C(I-G C)^{-1} R\right\|
$$

with $C$ satisfying the $\left(M_{j}, P_{i}\right)$ causality conditions. This problem can be solved by the method indicated in Section I-A.

\section{Conclusions}

In this paper, we presented the solution to the problem of optimal $l^{\infty}$ to $l^{\infty}$ disturbance rejection in periodic systems. The key observation was that we can obtain a finite number of linear constraints (functionals) on the allowable subspace of $K=U Q V$ to account for the causality condition on $Q(0)$ when considering the equivalent LTI problem. These functionals combined with the functionals of the unconstrained problem in [2], [3] characterize exactly the allowable subspace for $K$. Therefore, to obtain the solution, the same method of solution for the unconstrained problem can be applied yielding a tractable linear programming problem in the same manner as in [2], [3]. Also, we showed how we can obtain optimal compensators for MRSD systems using an identical method of solution to the one presented. Finally, we indicated that the problem of robust stabilization in periodic and multirate systems can be analyzed without introducing conservatism by considering the problem for the equivalent LTI system.

\section{APPENDIX}

Herein, we indicate how to find all matrices $B$ (of appropriate dimensions) such that there are real numbers $c_{i}$ with

$$
U_{o}^{T}(0) B V_{o}^{T}(0)=\sum_{i=1}^{r} c_{i} R_{i}(0)
$$

First, we consider the following problem (PA).

Let $M$ be a given matrix and $\left\{E_{i}\right\}_{i=1}^{I}$ be a given set of linearly independent matrices. Find all matrices $W$ such that

$$
M W=\sum_{i} c_{i} E_{i}
$$

for some real numbers $c_{i}$.

The solution to PA goes as follows.

Let $E_{i}$ be partitioned to column vectors as $E_{i}=$ $\left(e_{1}^{i}, e_{2}^{i}, \cdots, e_{n}^{i}\right)$ and $W=\left(w_{1}, w_{2}, \cdots, w_{n}\right)$. Also, define $P_{k}$ $=-\left(e_{k}^{1}, e_{k}^{2}, \cdots, e_{k}^{I}\right)$ for $k=1,2, \cdots, n$ and $c^{T}=$ $\left(c_{1}, c_{2}, \cdots, c_{I}\right)$. Then

$$
\left(\begin{array}{ll}
M & P_{k}
\end{array}\right)\left(\begin{array}{c}
w_{k} \\
c
\end{array}\right)=0
$$

Define $P=\left(\begin{array}{c}P_{1} \\ P_{2} \\ \vdots \\ P_{n}\end{array}\right)$ and $\tilde{w}=\left(\begin{array}{c}w_{1} \\ w_{2} \\ \vdots \\ w_{n}\end{array}\right)$. Let $N=\operatorname{diag}(M$, 
$M, \cdots, M) P$ ) then $N\left(\begin{array}{l}\tilde{w} \\ c\end{array}\right)=0$. Therefore, we can compute a basis for $W$ by computing a basis for the null space of the matrix $N$ which completes the solution to PA.

In view of the above construction if $C=B V_{o}^{T}(0)$ then we can compute a basis $\left\{C_{j}\right\}_{j=1}^{j_{C}}$ for the space

$$
S_{C}=\left\{C: U_{o}^{T}(0) C=\sum_{i=1}^{r} c_{i} R_{i}(0)\right\} .
$$

Then we can compute a basis $\left\{D_{j}\right\}_{j=1}^{j_{D}}$ for the space

$$
S_{D}=\left\{D: V_{o}(0) D \in \operatorname{span}\left(\left\{C_{j}\right\}_{j=1}^{j_{C}}\right)\right\}
$$

Now, by defining $j_{B}=j_{D}$ and $B_{j}=D_{j}^{T} j=1, \cdots, j_{B}$ we obtain the required basis for $S_{B}$.

\section{REFERENCES}

[1] H. Chapellat, M. Dahleh, and S. P. Bhattacharyya, "Optimal disturbance rejection for periodic systems," Tech. Rep. 89-019, Texas A\&M Univ., College Station, TX.

[2] M. A. Dahleh and J. B. Pearson, " $l$ optimal feedback controllers for MIMO discrete-time systems," IEEE Trans. Automat. Contr., vol. AC-32, Apr. 1987.

[3] - "Optimal rejection of persistent disturbances, robust stability, and mixed sensitivity minimization," IEEE Trans. Automat. Contr., vol. AC-33, pp. 722-731, Aug. 1988.

[4] M. A. Dahleh and Y. Ohta, "A necessary and sufficient condition for robust BIBO stability," Syst. Contr. Lett., vol. 11, pp. 271-275, 1988.

[5] C. A. Desoer and M. Vidyasagar, Feedback Systems: Input-Output Properties. New York: Academic, 1975.

[6] C. A. Desoer, R-W. Liu, J. Murray, and R. Saeks, "Feedback system design: The fractional representation approach to analysis and synthesis," IEEE Trans. Automat. Contr., vol. AC-25, no. 3, pp. 399-412, June 1980.

[7] B. A. Francis, $A$ Course in $H_{\infty}$ Control Theory. New York: Springer-Verlag, 1987.

[8] T. Kailath, Linear Systems. Englewood Cliffs, NJ: Prentice-Hall, 1980.

[9] P. P. Khargonekar, K. Poolla, and A. Tannenbaum, "Robust control of linear time-invariant plants using periodic compensation," IEEE Trans. Automat. Contr., vol. AC-30, no. 11, pp. 1088-1096, Nov. 1985.

[10] D. G. Luenberger, Optimization by Vector Space Methods. New York: Wiley, 1969.

[11] _ , " $l_{1}$-optimal control of multivariable systems with output norm constraints," Automatica, vol. 27, pp. 317-329, 1991.

[12] D. G. Meyer, "A new class of shift-varying operators, their shift-invariant equivalents, and multirate digital systems," IEEE Trans. Automat. Contr., vol. 35, pp. 429-433, Apr. 1990.

[13] _-, "A parametrization of stabilizing controllers for multirate sampled-data systems," IEEE Trans. Automat. Contr., vol. 35, no. 2, pp. 233-236, Feb. 1990.

[14] , "Performance robustness of discrete-time systems with structured uncertainty," IEEE Trans. Automat. Contr., vol. 36, pp. 398-412, Apr. 1991.

[15] R. Ravi, P. P. Khargonekar, K. D. Minto, and C. N. Nett, "Controller parametrization for time-varying multirate plants," IEEE Trans. Automat. Contr., vol. 35, no. 11, pp. 1259-1262, Nov. 1990.

[16] J. S. Shamma and M. A. Dahleh, "Time-varying versus time-invariant compensation for rejection of persistent bounded disturbance and robust stability," IEEE Trans. Automat. Contr., vol. 36, no. 7 , pp. 838-847, July 1991 .
[17] M. Vidyasagar, Control Systems Synthesis: A Factorization Approach. Cambridge, MA: M.I.T. Press, 1985

[18] D. C. Youla, H. A. Jabr, and J. J. Bongiorno, "Modern Wiener-Hopf design of optimal controllers-Part 2: The multivariable case,": IEEE Trans. Automat. Contr., vol. AC-21, June 1976.

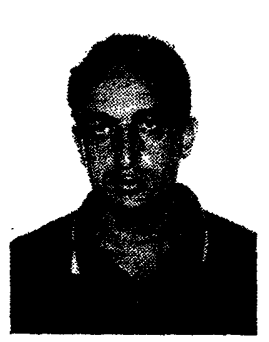

Munther A. Dahleh (S'84-M'87) was born in August 1962. He received the B.S.EE degree from Texas A\&M University, College Station, in 1983 and the Ph.D. degree from Rice University, Houston, TX, 1987.

Since 1987, he has been with the Department of Electrical Engineering and Computer Science, Massachusetts Institute of Technology, Cambridge, MA, where he is currently an Associate Professor. $\mathrm{He}$ has held consulting positions with NASA and the C.S. Draper Laboratory since 1988. His current interests include robust control, identification of uncertain systems and adaptation, and control of time-varying and infinite-dimensional systems.

Dr. Dahleh is currently serving as an Associate Editor for the Systems and Control Letters.

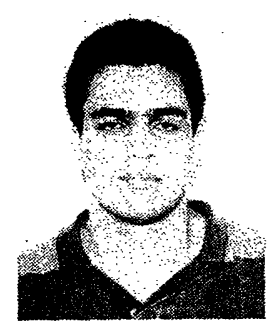

Petros G. Voulgaris was born in Athens, Greece, on January 28, 1963. He received the Diploma of Mechanical Engineering from the National Technical University of Athens in 1986, the S.M. and $\mathrm{Ph} . \mathrm{D}$. degrees in aeronautics and astronautics from the Massachusetts Institute of Technology, Cambridge, MA, in 1988 and 1991, respectively.

He has been Assistant Professor at the Department of Aeronautical and Astronautical Engineering, University of Illinois, Urbana-Champaign since August 1991. Research interests belong in the area of robust control for time-varying and nonlinear systems.

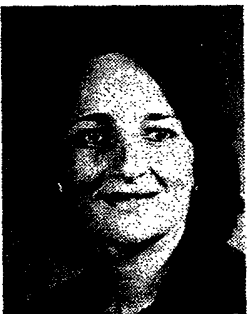

Lena S. Valavani (S'75-S'78-M'78) received the Ph.D. degree from the Department of Engineering and Applied Science, Yale University, New Haven, CT, in 1978.

She has been an Associate Professor in the Department of Aeronautics and Astronautics at the Massachusetts Institute of Technology since 1988. She was previousiy Boeing Assistant Professor of Aeronautics in the same department. Before joining the Department of Aeronautics and Astronautics she was a post-doctoral fellow at Yale and, subsequently, she held simultaneously a part-time appointment as a Research Associate at the Laboratory for Information and Decision Systems (LIDS) at M.I.T. and as a Staff Member at the C.S. Draper Laboratory until 1985. Her research interests lie in the areas of robust, nonlinear, and adaptive control and estimation/identification, and in the innovative application of control theory to complex engineering systems of special interest to aeronautics.

Dr. Valavani is a Member of Sigma Xi, IFAC, and an Associate Fellow of AIAA. She has been a consultant to the C.S. Draper Laboratory and, currently, to Lincoln Laboratory. She is an Associate Editor of Automatica and the International Journal for Nonlinear Control and Applications, a Past Associate Editor of IEEE Transactions on Automatic CONTROL, and has served as a Member of the National Research Council/Energy Engineering Board and of the National Science Foundation Review Board. 